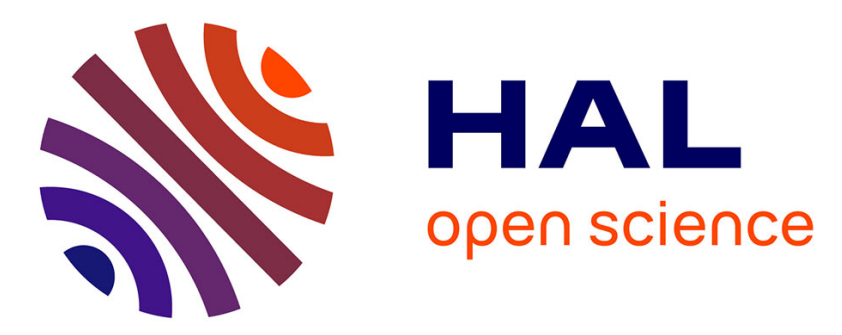

\title{
Efficient formation route of the prebiotic molecule formamide on interstellar dust grains
}

\author{
F. Dulieu, T. Nguyen, E. Congiu, S. Baouche, V. Taquet
}

\section{To cite this version:}

F. Dulieu, T. Nguyen, E. Congiu, S. Baouche, V. Taquet. Efficient formation route of the prebiotic molecule formamide on interstellar dust grains. Monthly Notices of the Royal Astronomical Society: Letters, 2019, 484 (1), pp.L119-L123. 10.1093/mnrasl/slz013 . hal-02389386

\section{HAL Id: hal-02389386 https://hal.science/hal-02389386}

Submitted on 2 Dec 2019

HAL is a multi-disciplinary open access archive for the deposit and dissemination of scientific research documents, whether they are published or not. The documents may come from teaching and research institutions in France or abroad, or from public or private research centers.
L'archive ouverte pluridisciplinaire HAL, est destinée au dépôt et à la diffusion de documents scientifiques de niveau recherche, publiés ou non, émanant des établissements d'enseignement et de recherche français ou étrangers, des laboratoires publics ou privés. 


\title{
Efficient formation route of the pre-biotic molecule formamide on interstellar dust grains
}

\author{
F. Dulieu, ${ }^{1 \star}$ T. Nguyen, ${ }^{1}$ E. Congiu, ${ }^{1}$ S. Baouche, ${ }^{1}$ and V. Taquet ${ }^{2}$ \\ ${ }^{1}$ Université de Cergy-Pontoise, Sorbonne Université, Observatoire de Paris, PSL University, CNRS, LERMA, F-95000, Cergy-Pontoise, France \\ ${ }^{2}$ Osservatorio Astrofisico di Arcetri, Largo E. Fermi 5, Firenze, 50125, Italy
}

Accepted XXX. Received YYY; in original form ZZZ

\begin{abstract}
Interstellar Complex Organic Molecules are thought to be the building blocks of more complex pre-biotic compounds. In particular, formamide (or methanimide, $\mathrm{NH}_{2} \mathrm{CHO}$ ), is presented as a multifunctional pre-biotic precursor, the starting point of both pregenetic and pre-metabolic species. $\mathrm{NH}_{2} \mathrm{CHO}$ is widely observed in different astrophysical media, as well as in comets that may have had a crucial role in the delivery of exogenous material to Earth. In star forming regions, gas phase synthesis of formamide is possible, even if it is still debated. In this paper, we present laboratory experiments demonstrating formamide formation in interstellar ice analogues at astronomically relevant temperatures via simultaneous hydrogenation of $\mathrm{NO}$ and $\mathrm{H}_{2} \mathrm{CO}$, two abundant molecules in star-forming regions. Inclusion of the experimental results in an astrochemical gas-grain model confirms the importance of the investigated solidstate reaction channel leading a high yield of formamide in dark interstellar clouds, and adds a valuable perspective about the way this refractory molecule may have been part of the pre-biotic molecular building blocks delivered to the young Earth.
\end{abstract}

Key words: Astrobiology-Astrochemistry- Molecular processes- methods: laboratory: solid state

\section{INTRODUCTION}

Among interstellar complex organic molecules (iCOMs), formamide has always attracted attention, since it is the prebiotic compound par excellence containing nitrogen, oxygen, carbon, and a peptide-like bond. Recently, it has been presented as the link in the chemical chain able to reconcile the "genetic first" and "metabolism first" paradigms (Saladino et al. 2012). Of all iCOMs, formamide has a very high binding energy, is known to survive on dust grains beyond sublimation of water (Urso et al. 2017; Chaabouni et al. 2018)âA ă and is therefore a very important molecule being also a source of interstellar nitrogen locked into a refractory animated species. Formamide has been detected in comet coma with a relatively low abundance (some $10^{-4}$, with respect to water) (Biver et al. 2014; Le Roy et al. 2015), while it is the second most abundant cometary soil compound ( $1.8 \%$ relative to water ice) as measured by the COSAC mass spectrometer on board the Philae lander that touched down on comet 67P/C-G (Goesmann et al. 2015)âĄă. There are three different possible scenarios to explain the presence of $\mathrm{NH}_{2} \mathrm{CHO}$ at rather high concentrations in the solid

^ E-mail: Francois.Dulieu@obspm.fr state, and all of them might have occurred at the time of the pre-solar nebula when pristine material from the origin of the solar system condensed and part of it remained locked up in comets (Mumma \& Charnley 2011)âĄă. The first scenario shows the formation of formamide only in the gas phase (Barone et al. 2015)âĄă, then its freeze-out onto dust grains. However, this hypothesis would lead to a small amount of formamide since its gas phase concentration is never high enough $\left(10^{-11}\right.$ relative to $\left.\mathrm{H}\right)$ even though gas phase production can be completed by solid phase production (Quénard et al. 2018). The second scenario implies the production of formamide via energetic processing of ice mantles (Gerakines et al. 2004; Jones et al. 2011; Urso et al. 2017)âĄă. That pathway to $\mathrm{NH}_{2} \mathrm{CHO}$ leads to a low but reasonable production rate, although it should be noted that several secondary products are synthesized at the same time (e.g., $\mathrm{HNCO}$ or $\mathrm{HC}_{2} \mathrm{O}_{3}$ ), depending on the initial composition of the icy mantle and the type of particle/energy employed (UV, ions, or electrons.). One experimental study also involves simultaneous UV irradiation and addition of $\mathrm{H}$ atoms onto mixtures of $\mathrm{NO}: \mathrm{CO}, \mathrm{NO}: \mathrm{H}_{2} \mathrm{CO}$ or $\mathrm{NO}: \mathrm{CH}_{3} \mathrm{OH}$ ices, which leads to a relatively high efficiency of $\mathrm{NH}_{2} \mathrm{CHO}$ production (Fedoseev et al. 2016). However, the initial ice composition used in those experiments may be quite differ- 
ent from the observed water-dominated interstellar icy dust grains and water can change dramatically the final product of UV irradiation or $\mathrm{H}$ exposure (Butscher et al. 2016; Watanabe et al. 2004). The third and last scenario of formamide formation implies its direct synthesis as soon as the molecular mantle covering dust grains is being formed. This is usually called the non-energetic pathway, because at this stage the temperature remains as low as the typical molecular cloud value $(\sim 10 \mathrm{~K})$, and no external energy source (except for chemical energy) is involved. Such conditions are found before the birth of a star has commenced. Conversely, within proto-solar nebulae the energy processes are highly variable, both locally and over time, due to the very complex mixing and dynamics of the surrounding matter. The aim of this letter is to investigate this last scenario by combining experiments and including their conclusions to current astrochemical models.

\section{EXPERIMENTAL RESULTS}

Our new experimental device VENUS ("VErs de NoUvelles Synthèses"), an ultrahigh vacuum (UHV) setup, allows us to simulate the cold molecular phase where interstellar ices are grown. A gold-coated OFHC copper substrate is placed in the centre of the UHV chamber (base pressure $10^{-10} \mathrm{mbar}$ ), and can be exposed to up to five atomic or molecular beams. The substrate is mounted in thermal contact with the cold finger of a closed-cycle $\mathrm{He}$ cryostat and its temperature is computer-controlled in the range 7-380 K. Deposited and newly formed species are monitored in situ by means of Fourier Transform Reflection Absorption InfraRed Spectroscopy (RAIRS) using vibrational fingerprint spectra in the $4000-700 \mathrm{~cm}^{-1}$ region and in the gas phase by means of Quadrupole Mass Spectrometry (QMS) upon temperature-programmed desorption (TPD) by steadily heating the $\mathrm{Cu}$ substrate (by $0.2 \mathrm{~K} / \mathrm{s}$ ) and monitoring evaporated species. The beams have fluxes in the order of $2 \times 10^{12}$ molecules $/ \mathrm{cm}^{2} / \mathrm{s}$, which means that one monolayer ( $1 \mathrm{ML}$, corresponding to $10^{15}$ molecules $/ \mathrm{cm}^{2}$ ) is deposited in about 10 minutes. This also means that a molecule already sitting on the surface will wait on average 10 minutes before another incoming particle from the beam hits it or sticks on top of it. Under these conditions, surface diffusion processes (Langmuir-Hinshelwood mechanism) are preferred over gas-surface interactions (Eley-Rideal type).

Figure 1 shows the integrated area of the NO dimer bands at 1774 and $1865 \mathrm{~cm}^{-1}$ (red stars) and the $\mathrm{H}_{2} \mathrm{CO}$ band at $1730 \mathrm{~cm}^{-1}$ (blue squares) when $\mathrm{NO}$ and $\mathrm{H}_{2} \mathrm{CO}$ separately deposited on the surface maintained at $10 \mathrm{~K}$. The same result is obtained when the two molecules are deposited simultaneously, like in a co-deposition experiment. Despite the dispersion of the signal due to the small number of molecules deposited (about $10^{14}$ in total, i.e. less than one nanomole), there is a linear increase in absorbance with exposure, as expected. Brown squares and brown stars represent the integrated area of the $\mathrm{H}_{2} \mathrm{CO}$ and $\mathrm{NO}$ bands, respectively, measured during a co-deposition of $\mathrm{H}_{2} \mathrm{CO}$, $\mathrm{NO}$ and $\mathrm{H}$ atoms. The brown symbols are below noise level which indicates that all $\mathrm{H}_{2} \mathrm{CO}$ and $\mathrm{NO}$ molecules reacted during exposure. Therefore, the reactants are completely consumed

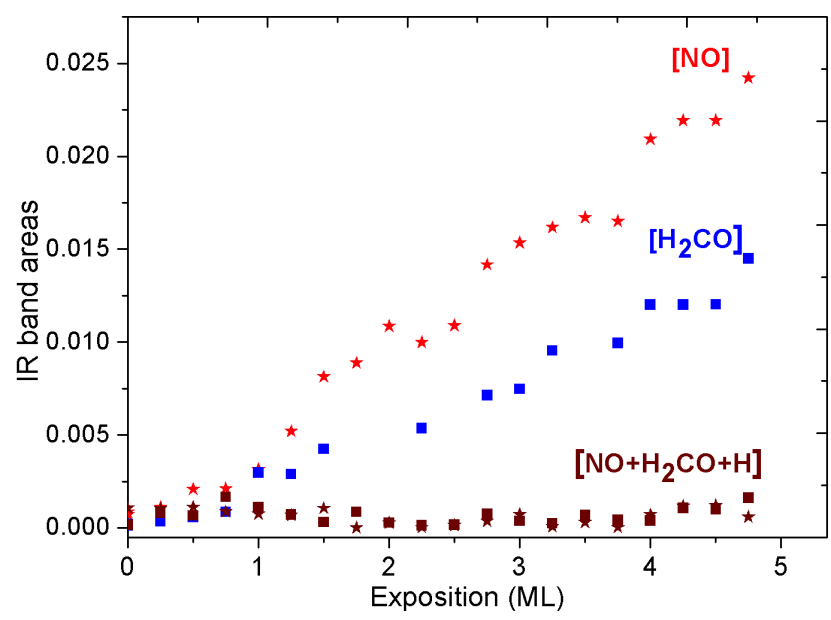

Figure 1. Integrated areas of $\mathrm{NO}$ band (stars) and $\mathrm{H}_{2} \mathrm{CO}$ band (squares) as a function of deposited dose on the gold-coated substrate held at $10 \mathrm{~K}$. Blue and red symbols are obtained in pure $\mathrm{H}_{2} \mathrm{CO}$ and $\mathrm{NO}$ experiments, and brown symbols are obtained in the $\mathrm{NO}+\mathrm{H}_{2} \mathrm{CO}+\mathrm{H}$ (co-deposition) experiment.

at $10 \mathrm{~K}$, suggesting that all reactions occur at such a low temperature.

In very thin layers of adsorbates and under the continuous addition of $\mathrm{H}$ atoms, very few radicals should remain at the end of the exposure phase. In fact, the flux of $\mathrm{H}$ atoms is about 4 times more intense than either $\mathrm{H}_{2} \mathrm{CO}$ or NO flux, and under these conditions the complete hydrogenation of $\mathrm{NO}$ or $\mathrm{H}_{2} \mathrm{CO}$ alone is consistent with previous works (Congiu et al. 2012a; Minissale et al. 2016a)âĄă. We have chosen this $\mathrm{H}$ flux to be sufficient to keep the signal of NO dimers undetectable so it can be compared to astrophysical conditions, where NO dimers are not supposed to play a role. We know that hydrogenation of $\mathrm{H}_{2} \mathrm{CO}$ produces both $\mathrm{CO}$ by abstraction of protons and $\mathrm{CH}_{3} \mathrm{OH}$ by sequential addition of $\mathrm{H}$ atoms (Hidaka et al. 2007; Hiraoka et al. 2005; Minissale et al. 2016a)âĄă, which allows us to assume that $\mathrm{HCO}$ and $\mathrm{CH}_{3} \mathrm{O}$ radicals are transiently produced whenever both $\mathrm{H}_{2} \mathrm{CO}$ and $\mathrm{H}$ are deposited on the cold surface. The other reactive channel is the hydrogenation of $\mathrm{NO}$, which leads to hydroxylamine $\left(\mathrm{NH}_{2} \mathrm{OH}\right)$ (Congiu et al. 2012b), and if some dimers of $\mathrm{NO}$ are present on the surfaces, is also a source of $\mathrm{N}_{2} \mathrm{O}$ and $\mathrm{H}_{2} \mathrm{O}$ in small amounts Congiu et al. (2012a)âA ă. It would be necessary to add the possible role of $\mathrm{H}_{2} \mathrm{NO}$ and $\mathrm{OH}$ to the list of radicals. However, in our experiments co-deposition of all reactants is used. NO is readily consumed and NO dimers are unlikely to be present at any time, therefore it is fair to assume that $\mathrm{H}_{2} \mathrm{NO}$ remains the only active radical.

Figure 2 shows the TPD profiles of the co-deposition experiment of $\left\{\mathrm{NO}+\mathrm{D}_{2} \mathrm{CO}+\mathrm{D}\right\}$ at $10 \mathrm{~K}$ on the gold-coated surface (top panel). Deuterated reactants were used in this case to cross check the mass of the newly formed species as to ensure that the final products are chemically identical though they have masses corresponding to the deuterated forms. In the bottom panel of Figure 2 we show the TPD profiles obtained after co-deposition of $\left\{\mathrm{NO}+\mathrm{H}_{2} \mathrm{CO}+\mathrm{H}\right\}$ on $4 \mathrm{ML}$ of previously deposited amorphous solid water ice (ASW) held at $10 \mathrm{~K}$. We first grow a $4 \mathrm{ML}$ thick amor- 


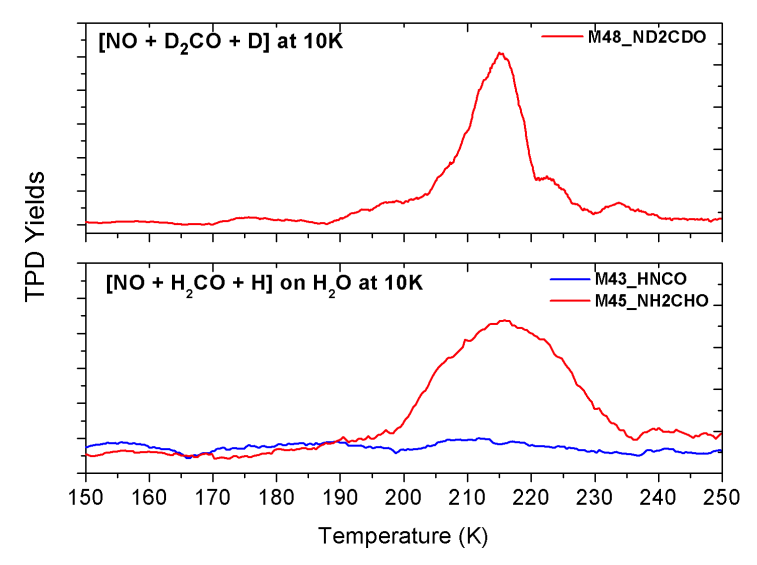

Figure 2. TPD profiles obtained after co-depostion of $\left\{\mathrm{NO}+\mathrm{D}_{2} \mathrm{CO}+\mathrm{D}\right\}$ on gold held at $10 \mathrm{~K}$ (top panel), and TPD profiles after co-deposition of $\left\{\mathrm{NO}+\mathrm{H}_{2} \mathrm{CO}+\mathrm{H}\right\}$ on compact ASW ice at $10 \mathrm{~K}$ (bottom panel).

phous water ice substrate at $10 \mathrm{~K}$ before commencing the codeposition of all reactants. It should be noted that ASW ice is porous when deposited at $10 \mathrm{~K}$. This experiment is carried out under conditions that more realistically mimic interstellar ices where molecular complexity develops on dust grains, and where ASW is the dominant solid species (Boogert et al. 2015)âĄă. The top panel of Figure 2 shows the mass profile for $\mathrm{m} / \mathrm{z}=48$, corresponding to $\mathrm{ND}_{2} \mathrm{CDO}$, (deuterated formamide). Formamide is also accompanied by the desorption of $\mathrm{CD}_{3} \mathrm{OD}$ (deuterated methanol) and $\mathrm{ND}_{2} \mathrm{OD}$ (deuterated hydroxylamine). The lower panel shows the desorption profiles for $\mathrm{m} / \mathrm{z}=45\left(\mathrm{NH}_{2} \mathrm{CHO}\right)$ and $\mathrm{m} / \mathrm{z}=43(\mathrm{HNCO})$. Given i) the high desorption temperature (above $210 \mathrm{~K}$ ) of the mass spectrum peak for $\mathrm{m} / \mathrm{z}=45$, ii) the unambiguous identification of the fragments induced in the QMS head upon ionization and iii) the correct value of the masses in the deuterated-species experiment, there is no doubt that formamide was synthesized in our experiments. Also, we determined that $\mathrm{HNCO}$ was not produced at all (Figure 2, bottom panel).

In the additional material we show the TPD profiles of methanol $(\mathrm{m} / \mathrm{z}=32$, desorption peak at around $150 \mathrm{~K})$, $\mathrm{NH}_{2} \mathrm{OH}(\mathrm{m} / \mathrm{z}=33$, peak at $180 \mathrm{~K})$ and $\mathrm{NH}_{2} \mathrm{CHO}(\mathrm{m} / \mathrm{z}=45$, peat at $215 \mathrm{~K}$ ). From these mass spectra and from the fragments mass profiles (such as for $\mathrm{m} / \mathrm{z}=29,30,31$ ) integrated over the appropriate desorption temperature range, it is possible to measure the relative ratios of the three major products, namely methanol, hydroxylamine, and formamide. In the pie diagrams displayed in Figure 3 we show the relative abundance of products obtained in two key experiments. The product ratios change considerably in the presence of water molecules. An analysis of these ratios in the light of the different chemical pathways shows that hydrogenation of $\mathrm{NO}$ is the most effective reactive subsystem as proven by the high $\mathrm{NH}_{2} \mathrm{OH}$ formation rate. This is because its first reactive step $(\mathrm{NO}+\mathrm{H} \rightarrow \mathrm{HNO})$ has a very low or no energy barrier (Congiu et al. 2012b)âĄă, whereas hydrogenation of $\mathrm{H}_{2} \mathrm{CO}$ possesses a small activation barrier that can only be overcome by quantum tunnelling effect at $10 \mathrm{~K}$ (Hidaka et al. 2013)âĄă. In addition, a small fraction of $\mathrm{H}_{2} \mathrm{CO}$ can also be transformed into $\mathrm{CO}$, which eventually reduces

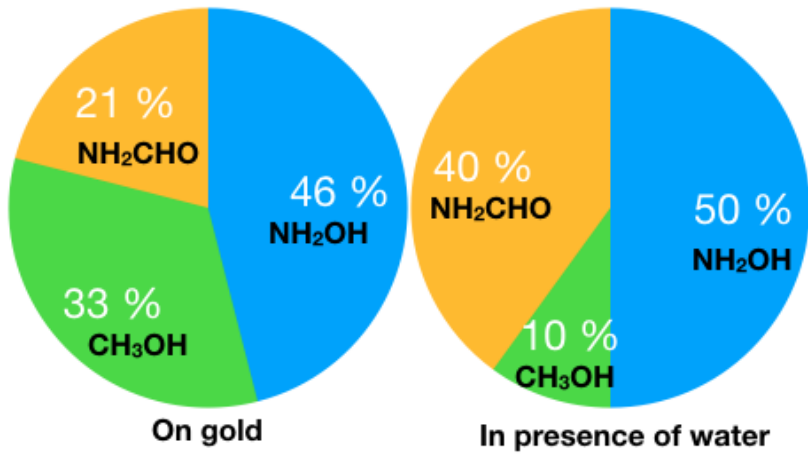

Figure 3. Relative abundance of products obtained from codeposition of $\mathrm{NO}, \mathrm{H}_{2} \mathrm{CO}$, and $\mathrm{H}$ on bare gold and on compact water ice at $10 \mathrm{~K}$.

the $\mathrm{CH}_{3} \mathrm{OH}$ production. Part of the newly formed species $(<10 \%)$ may also be lost via chemical desorption (Dulieu et al. 2013; Minissale et al. 2016b).

Without water molecules, only one-third of the $\mathrm{H}_{2} \mathrm{CO}$ is diverted from its hydrogenation pathway leading to methanol, and contributes to the formation of formamide. In the presence of water, most $\mathrm{H}_{2} \mathrm{CO}$ is converted into $\mathrm{NH}_{2} \mathrm{CHO}$, which eventually becomes four times more abundant than methanol. These simple experiments demonstrate that formamide is naturally formed following the co-hydrogenation $\mathrm{NO}$ and $\mathrm{H}_{2} \mathrm{CO}$, two relatively abundant iCOM precursors found in icy mantles covering cosmic dust grains.

\section{ASTROCHEMICAL MODEL}

To prove the impact of the new chemical pathway presented in this work and leading to $\mathrm{NH}_{2} \mathrm{CHO}$ in the solid phase, we use a standard astrochemical model that includes gasgrain chemical networks (Taquet et al. 2012; Taquet et al. 2014)âĄă. A complete study of the physical-chemical evolution from translucent dark clouds to protostars is beyond the scope of this work and would require a coupling with a detailed physical model. Instead, we use here a pseudo-time dependent approach where physical conditions are constant and are chosen as in typical molecular clouds: $\mathrm{n}_{H}=10^{4} \mathrm{~cm}^{-3}$, $\mathrm{A}_{v}=20 \mathrm{mag}, \zeta=10^{-17} \mathrm{~s}^{-1}$, and an equilibrium temperature of gas and grains of $10 \mathrm{~K}$.Briefly, the GRAINOBLE model follows the temporal evolution of chemical abundances in the gas phase and in interstellar ices. We use the rate equations approach developed by Hasegawa \& Herbst (1993) to follow the formation of interstellar ices by considering three sets of differential equations: one for gas-phase species, one for surface species, and one for solid species in the icy mantle. The equations governing chemical abundances on the surface and in the bulk are linked by an additional term that is proportional to the rate of growth or evaporation of the grain mantle. Surface species are continuously trapped into the bulk because of the accretion of new particles. The model considers five main gas-grain surface processes: i) accretion of gas-phase species on the surface of spherical grains with a constant size; ii) diffusion of adsorbed species via thermal hopping; iii) reaction between two particles at the ice surface 
and in the ice mantle; iv) desorption of adsorbed species into the gas phase through several thermal evaporation as well as chemical desorption, and cosmic-ray induced desorption; v) photo-dissociation and photo-evaporation induced by the interstellar and secondary UV radiation fields. We assume all elements to be initially in the atomic form in the gas phase, except for $\mathrm{H}$ that is assumed to be already converted into $\mathrm{H}_{2}$ given the efficient $\mathrm{H}-\mathrm{H}_{2}$ conversion in dark clouds at low visual extinctions. The elemental abundances correspond to the set EA1 of Wakelam \& Herbst (2008): $X\left(\mathrm{H}_{2}\right)$ $=0.5, X(\mathrm{H})=5 \times 10^{-5}, X(\mathrm{He})=0.09, X(\mathrm{C})=7.30 \times 10^{-5}$, $X(\mathrm{~N})=2.14 \times 10^{-5}, X(\mathrm{O})=1.76 \times 10^{-4}, X(\mathrm{Si})=8.0 \times 10^{-9}$, $X(\mathrm{~S})=8.0 \times 10^{-8}, X(\mathrm{Fe})=3.0 \times 10^{-9}, X(\mathrm{Na})=2.0 \times 10^{-9}$, $X(\mathrm{Mg})=7.0 \times 10^{-9}, X(\mathrm{Cl})=1.0 \times 10^{-9}$. The gas-grain chemical network is the one presented in Taquet et al. (2016)âA ă. The gas-phase chemical network is based on the 2013 version of the Kinetic Database for Astrochemistry (KIDA) chemical database Wakelam et al. (2012)âĄă. It has been further updated to include warm gas-phase chemistry involving water and ion-neutral reactions involving ozone. The surface network includes the chemistry of all dominant ice components $\left(\mathrm{H}_{2} \mathrm{O}, \mathrm{CO}, \mathrm{CO}_{2}, \mathrm{NH}_{3}, \mathrm{CH}_{4}, \mathrm{H}_{2} \mathrm{CO}\right.$, and $\left.\mathrm{CH}_{3} \mathrm{OH}\right)$, as well as iCOMs. We have added the following reaction to the solid-phase chemical network:

$\mathrm{H}_{2} \mathrm{NO}+\mathrm{H}_{2} \mathrm{CO} \rightarrow \mathrm{NH}_{2} \mathrm{CHO}+\mathrm{OH}$

and the complete list of reactions involving NO and its subsequent hydrogenated forms on grains, based on Ioppolo et al. (2015). Furthermore, we have added the possibility of abstraction of protons from $\mathrm{NH}_{2} \mathrm{OH}$ :

$\mathrm{NH}_{2} \mathrm{OH}+\mathrm{H} \rightarrow \mathrm{H}_{2} \mathrm{NO}+\mathrm{H}_{2}$,

which may be a key reaction since it contributes to the increase in $\mathrm{H}_{2} \mathrm{NO}$, a precursor of formamide. This specific point has been tested experimentally and will be addressed in a forthcoming publication. In the rate equations formalism introduced by Hasegawa \& Herbst (1992)âĄă, accreted species can react when they meet with each other in the same site by the Langmuir-Hinshelwood mechanism. The reaction rate is governed by the diffusion of at least one mobile reactant for the low (i.e. $T \sim 10 \mathrm{~K}$ ) temperatures considered in this work. Here at $T \sim 10 \mathrm{~K}$, the formamide formation is triggered by a reaction that involves two heavy molecules that do not diffuse along the surface or within the ice mantle. However, one of the two reactants, $\mathrm{H}_{2} \mathrm{CO}$, is expected to be relatively abundant in interstellar ices and the two reactants have a certain probability to be neighbour molecules which is simply given by the abundance of $\mathrm{H}_{2} \mathrm{CO}$ in the ices. In practice, we followed the formation of two types of $\mathrm{H}_{2} \mathrm{NO}$ molecules: those that are formed next to an active $\mathrm{H}_{2} \mathrm{CO}$ molecule available to react and those that are formed next to other molecules. For all chemical processes forming $\mathrm{H}_{2} \mathrm{NO}$, two outcomes are introduced with a branching ratio given by the abundance of $\mathrm{H}_{2} \mathrm{CO}$ molecules. The rate of the reaction between $\mathrm{H}_{2} \mathrm{NO}$ and active $\mathrm{H}_{2} \mathrm{CO}$ is therefore given by the product between the maximal vibrational frequency of the two reactants $v_{0}$ and the probability of the reaction $P_{\text {reac. }}$. In experiments as well as in the dense interstellar medium, $\mathrm{NO}$ and $\mathrm{H}_{2} \mathrm{CO}$ must be neighbours to react together since they are not mobile at $10 \mathrm{~K}$. Our experimental observations confirm that the barrier-less hydrogenation of NO leads to reaction 1 more efficiently than the hydro- genation of $\mathrm{H}_{2} \mathrm{CO}$, which activates reaction 1 and makes it the dominant chemical route consuming $\mathrm{H}_{2} \mathrm{CO}$ molecules. In the model, we did not consider other possible reactions such as $\mathrm{CH}_{3} \mathrm{O}+\mathrm{HNO}$, because it is not efficient under our experimental conditions. In any case, whatever the details of the actual chemical network may be, the proximity of $\mathrm{H}_{2} \mathrm{CO}$ and NO molecules on the surface represents the limiting factor in astrochemical models and as such it has to be evaluated according to the molecular abundances on cosmic grains. Figure 4 shows the absolute abundance of the main solid species and the abundance of $\mathrm{NO}, \mathrm{NH}_{2} \mathrm{CHO}, \mathrm{CH}_{3} \mathrm{OH}$ and $\mathrm{N}_{2} \mathrm{O}$ ices relative to water, as function of time in our simulation. Dashed lines represent the solution assuming a high barrier $(1000 \mathrm{~K})$ to reaction 2 , whereas the reaction 2 is supposed to be barrier-less in in solid lines. The time needed for growing a molecular layer on a grain is typically $10^{4}$ years, and a few million years is the time-scale employed by interstellar icy mantles to reach their final thickness of a few hundred layers. Therefore, the predicted abundances at one million years represent the molecular composition of the ice at the onset of the formation of a solar system. After this time, there are almost no more reactive gas-phase species that can accrete on grains. In Figure 4, we observe a production of $\mathrm{NH}_{2} \mathrm{CHO}$ of about $10^{-4}$ relative to water. In the case of instant gas release from the mantle due to sputtering, as in the case of shocks, this would produce a gas phase abundance of some $10^{-8}$ relative to $\mathrm{H}_{2}$, which is slightly higher, but not inconsistent given the uncertainties in the reaction rates and in the observations, than the observed values of 0.4-1.1 $\times 10^{-8}$ (López-Sepulcre et al. 2015). Moreover, the abundances could evolve given that the sublimation of ices would be followed by gas phase chemistry that, in its turn, would modify the abundance ratios. The model accounts for an effective reduction of $\mathrm{NH}_{2} \mathrm{OH}$ that, though it is a direct product of hydrogenation of $\mathrm{NO}$ on grains, remains an elusive species in space (Codella et al. 2018) and is for this reason an unsolved problem in today's astrochemistry. In the case of a relatively low or no energy barrier for $\mathrm{H}$-abstraction of $\mathrm{NH}_{2} \mathrm{OH}$, hydroxylamine would be consumed and formamide would be formed at the surface of the dust grains, as shown in the right panel of Figure 4. As opposed to what we observe in the laboratory, we find that methanol production is not affected in the model. The different results concerning methanol can be explained by the great proximity between $\mathrm{H}_{2} \mathrm{CO}$ and $\mathrm{NO}$ molecules in the experiments, during the deposition phase, which favours formamide production and eventually becomes the dominant process. In space, abundances are much weaker and NO is less abundant than $\mathrm{H}_{2} \mathrm{CO}$ so most $\mathrm{H}_{2} \mathrm{CO}$ undergoes a series of hydrogenation steps that lead to methanol.

Despite very different fluxes and proportions of reagents under our experimental conditions and in space, the limiting factor for formamide synthesis is the proximity of $\mathrm{NO}$ and $\mathrm{H}_{2} \mathrm{CO}$. In the laboratory and in the interstellar medium hydrogenation is very effective, so formamide should be synthesized in both cases. However, if other chemical mechanisms slower than one minute (experimental time) but faster than one day (astrophysical rate of $\mathrm{H}$ adsorption) are at play, or if also other abundant chemical neighbours (such as CO or $\mathrm{H}_{2}$ ) become reaction partners, our conclusions could be too straightforward and this would require adequate changes in the model. 

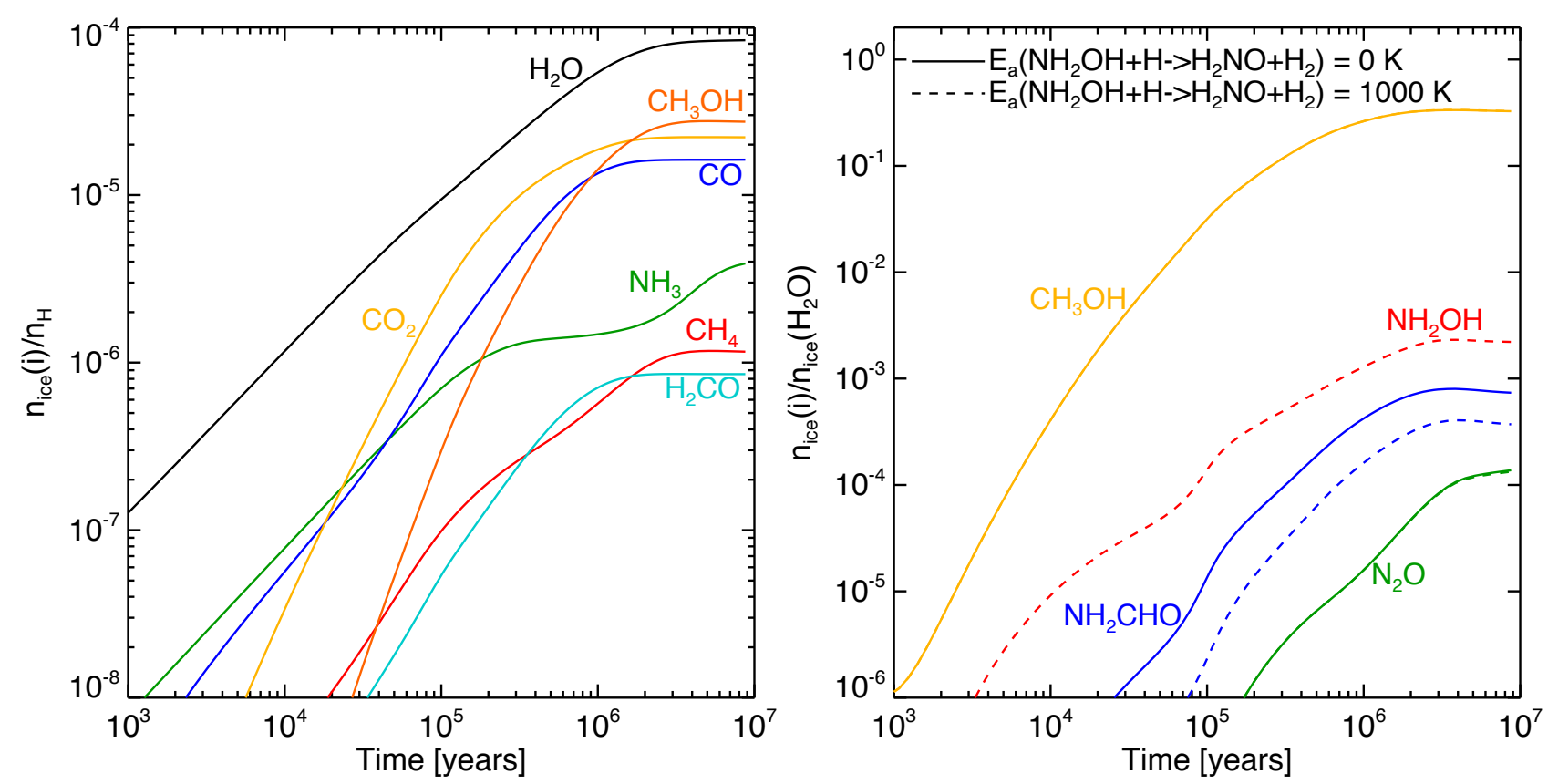

Figure 4. Absolute abundances of main solid species (left panel) and fractional abundances of solid species related to $\mathrm{NH}_{2} \mathrm{CHO}$ relative to water ice (right panel) as predicted by the GRAINOBLE astrochemical model with no activation barrier (solid lines) and for an activation barrier of $1000 \mathrm{~K}$ (dashed lines) for the $\mathrm{H}$-abstraction reaction of $\mathrm{NH}_{2} \mathrm{OH}$.

\section{CONCLUSIONS}

Taking into account the very low volatility of formamide (Chaabouni et al. 2018) and the efficient mechanism to form this pre-biotic molecule in the solid state as presented in this work, allows us to reconcile observations of a high amount of formamide in material sampled by COSAC at the comet $67 \mathrm{P}$ surface and its low gas phase abundance. In addition, the relative low abundance of HNCO measured in cometary soil corroborates a solid state formation route to formamide decoupled from HNCO formation. Our work shows that the simple hydrogenation of two abundant molecular precursor molecules in the interstellar medium leads to the formation of formamide, a key molecule in pre-biotic chemistry. Formamide is produced more efficiently than methanol under our experimental conditions. In dark molecular clouds, this chemical pathway would produce $\mathrm{NH}_{2} \mathrm{CHO}$ on grains with an abundance of about $10^{-4}$ relative to water, which is insufficient to allow detection of this species by current IR space telescopes. We expect, however, that in the future the next generation instruments with increased capabilities, such as the James Webb space telescope, may detect a faint absorption signature of $\mathrm{NH}_{2} \mathrm{CHO}$ ice. Furthermore, the existence of an effective and direct mechanism of formation of formamide in the solid state, without the need of energetic processes, supports the argument that cometary materials have strong and direct chemical links with their interstellar matrix, the pre-solar nebula. Comets that collide with planets would contribute to the delivery of exogenous materials, and the same mechanism might have contributed to bring about life on Earth. Some authors rely on the high degree of deuteration found in our oceans and that found in comets to determine if comets have been an essential contribution to the Earth's water reservoir. If this is confirmed, of all the species that comprise cometary bulks and have been locked up at the time of the early solar system, formamide is the most likely to withstand thermal desorption. Therefore, formamide is to be considered a key molecule for the early development of life, and its origin is to be found at the heart of dark clouds, on the surface of dust grains synthesized at temperatures as low as $10 \mathrm{~K}$, well before the formation of our own planet Earth.

\section{ACKNOWLEDGEMENTS}

TN thanks the Labex Michem program for her PHD grant. This work was supported by the Programme National "Physique et Chimie du Milieu Interstellaire" (PCMI) of CNRS/INSU with INC/INP co-funded by CEA and CNES, and by the DIM-ACAV+, a funding program of the Region Ile de France. V.T. acknowledges the financial support from the European Union's Horizon 2020 research and innovation programme under lhe Marie Sklodowska-Curie grant agreement n. 664931, and the Institute of Advanced Studies of the University of Cergy Pontoise.

\section{REFERENCES}

Barone V., Latouche C., Skouteris D., Vazart F., Balucani N., Ceccarelli C., Lefloch B., 2015, MNRAS, 453, L31

Biver N., et al., 2014, A\&A, 566, L5

Boogert A. A., Gerakines P. A., Whittet D. C., 2015, Annual Review of Astronomy and Astrophysics, 53, 541

Butscher T., Duvernay F., Danger G., Chiavassa T., 2016, Astronomy \& Astrophysics, 593, A60

Chaabouni H., Diana S., Nguyen T., Dulieu F., 2018, A\&A, 612, A47

Codella C., et al., 2018, MNRAS, 474, 5694 
Congiu E., Chaabouni H., Laffon C., Parent P., Baouche S., Dulieu F., 2012a, The Journal of chemical physics, 137, 054713 Congiu E., et al., 2012b, ApJ, 750, L12

Dulieu F., Congiu E., Noble J., Baouche S., Chaabouni H., Moudens A., Minissale M., Cazaux S., 2013, Scientific Reports, 3, 1338

Fedoseev G., Chuang K.-J., van Dishoeck E. F., Ioppolo S., Linnartz H., 2016, MNRAS, 460, 4297

Gerakines P. A., Moore M. H., Hudson R. L., 2004, Icarus, 170, 202

Goesmann F., et al., 2015, Science, 349, aab0689

Hasegawa T. I., Herbst E., 1992, in American Astronomical Society Meeting Abstracts \#180. p. 797

Hasegawa T. I., Herbst E., 1993, MNRAS, 263, 589

Hidaka H., Kouchi A., Watanabe N., 2007, The Journal of Chemical Physics, 126, 204707

Hidaka H., Watanabe M., Kouchi A., Watanabe N., 2013, in AIP Conference Proceedings. pp 318-325

Hiraoka K., et al., 2005, ApJ, 620, 542

Ioppolo S., McGuire B. A., de Vries X., Carroll B., Allodi M., Blake G., 2015, IAU General Assembly, 22, 2257519

Jones B. M., Bennett C. J., Kaiser R. I., 2011, ApJ, 734, 78

Le Roy L., et al., 2015, A\&A, 583, A1

López-Sepulcre A., et al., 2015, Monthly Notices of the Royal Astronomical Society, 449, 2438

Minissale M., Moudens A., Baouche S., Chaabouni H., Dulieu F., 2016a, Monthly Notices of the Royal Astronomical Society, 458, 2953

Minissale M., Dulieu F., Cazaux S., Hocuk S., 2016b, A\&A, 585, A24

Mumma M. J., Charnley S. B., 2011, Annual Review of Astronomy and Astrophysics, 49, 471

Quénard D., Jiménez-Serra I., Viti S., Holdship J., Coutens A., 2018, MNRAS, 474, 2796

Saladino R., Crestini C., Pino S., Costanzo G., Di Mauro E., 2012, Physics of life reviews, 9, 84

Taquet V., Ceccarelli C., Kahane C., 2012, A\&A, 538, A42

Taquet V., Charnley S. B., Sipilä O., 2014, The Astrophysical Journal, 791, 1

Taquet V., Furuya K., Walsh C., van Dishoeck E. F., 2016, MNRAS, 462, S99

Urso A. C., Chojnacki M., McEwen A., Dundas C., 2017, LPI Contributions, 1961, 3059

Wakelam V., Herbst E., 2008, ApJ, 680, 371

Wakelam V., et al., 2012, ApJS, 199, 21

Watanabe N., Nagaoka A., Shiraki T., Kouchi A., 2004, The Astrophysical Journal, 616, 638

\section{APPENDIX A: ADDITIONAL MATERIAL}

The figure A1 is an example of TPD curves from which the figure 3 has been made. Methanol is firstly desorbing at around $150 \mathrm{~K}$, co-desorbing with the water substrate. Hydroxylamine is mostly desorbing between 160 and $210 \mathrm{~K}$, and formamide desorption is peaking at $220 \mathrm{~K}$.

This paper has been typeset from a $\mathrm{T}_{\mathrm{E}} \mathrm{X} / \mathrm{L} \mathrm{LT}_{\mathrm{E}} \mathrm{X}$ file prepared by the author.

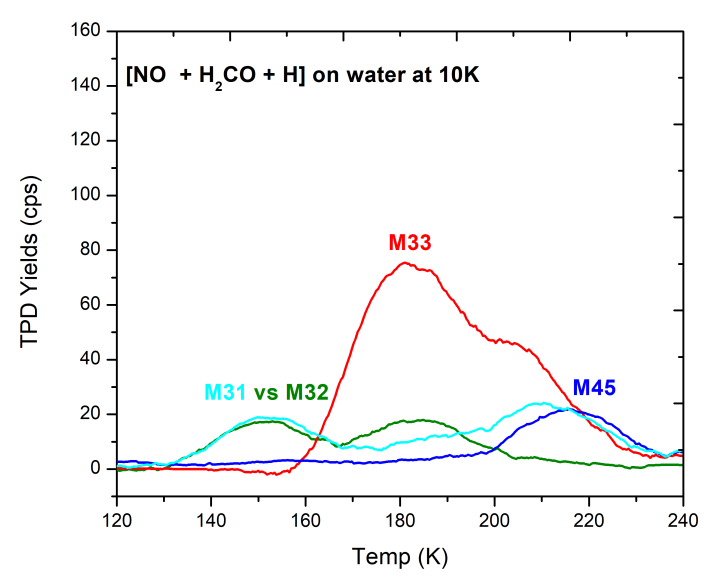

Figure A1. TPD profiles of methanol $(\mathrm{m} / \mathrm{z}=32), \mathrm{NH}_{2} \mathrm{OH}$ $(\mathrm{m} / \mathrm{z}=33)$, and $\mathrm{NH}_{2} \mathrm{CHO}(\mathrm{m} / \mathrm{z}=45)$ after co-deposition of $\left\{\mathrm{NO}+\mathrm{H}_{2} \mathrm{CO}+\mathrm{H}\right\}$ on $\mathrm{ASW}$ ice at $10 \mathrm{~K}$. 